\title{
ACTITUDES DE UNIVERSITARIOS HACIA LA ESTADÍSTICA COMO MATERIA DE ESTUDIO Y HERRAMIENTA PARA ANALIZAR DATOS
}

\author{
Siufong Acón Araya ${ }^{\text {a }}$ (D), \& Marianela Salazar Ramos ${ }^{b}{ }^{(i)}$ \\ Universidad Latina de Costa Rica, San José, Costa Rica. ${ }^{\text {a }}$ \\ Universidad Americana, San José, Costa Rica. ${ }^{\text {b }}$
}

\begin{abstract}
RESUMEN
Se analizan las actitudes hacia las estadísticas de estudiantes de una universidad privada en Costa Rica, según los componentes sociales, educativos, instrumentales, afectivos, cognitivos y comportamentales mediante una metodología no experimental y alcance descriptivo. Las actitudes se midieron mediante la Escala de Estrada (2002), en una muestra de 303 estudiantes de las Facultades de Ciencias Exactas, Ciencias de la Salud y Ciencias Sociales de la Universidad Latina de Costa Rica. Se determina que las dimensiones con puntajes mayores en la percepción de la estadística, son la afectiva y comportamental. Las actitudes favorables hacia la estadística se presentan mayormente en estudiantes de Ciencias Exactas, pese a que la generalidad del estudiantado no evita información con contenido estadístico para comprender situaciones académicas y sociales.
\end{abstract}

Palabras Claves

actitudes; estadística; universitarios; análisis de datos

\begin{abstract}
This study analyses the attitudes towards the statistics of students of a private university in Costa Rica, according to the social, educational, instrumental, affective, cognitive and behavioral components through a non-experimental methodology and descriptive scope. The attitudes were measured using the Estrada Scale (2002), in a sample of 303 students from the Faculties of Exact Sciences, Health Sciences and Social Sciences of the Universidad Latina de Costa Rica. It was determined that the dimensions with higher scores in the perception of statistics are affective and behavioral. Favorable attitudes towards statistics occur mostly in students of Exact Sciences, although the majority of students does not avoid information with statistical content to understand academic and social situations.
\end{abstract}

Keywords

attitudes, statistics, university students, data analysis

\footnotetext{
${ }^{1}$ Correspondence about this article should be addressed to Siufong Acón Araya: $\underline{\text { siufong.acon@ulatina.cr }}$
} 


\section{ATTITUDES TOWARDS STATISTICS AS STUDY MATTER AND TOOL FOR DATA ANALYSIS}

\section{Introducción}

La estadística como materia de estudio, se encuentra en numerosas mallas curriculares de carreras universitarias. En los últimos años el Ministerio de Educación Pública de Costa Rica (MEP) le ha dado énfasis a la enseñanza de la estadística tanto en primaria como secundaria (Ministerio de Educación Pública, 2017). Por tanto, los estudiantes que se graduaron en el 2016 de secundaria ya han adquirido conocimientos básicos que se ven implicados en los contenidos de esta materia, ya que los programas de III Ciclo incluyen temas asociados con la recolección, ordenamiento y análisis de datos, siendo esta la base de la estadística.

Empero, en el contexto universitario se ha evidenciado que independientemente del año de graduación de los estudiantes, las actitudes hacia la estadística varían, así como los conocimientos previos que se adquieren en la educación secundaria, ya que no siempre están presentes. Tal es el caso de investigaciones desarrolladas en áreas atinentes a las Ciencias Sociales, como educación, en donde se comprueba que las experiencias previas en su proceso formativo y los conocimientos que se tengan en estadística, tienden a evocar en algunos casos reacciones desfavorables en los estudiantes (Covadonga, 2015). Ahora bien, las bases que han fortalecido la formación en estadística, contribuyen notablemente en el proceso de aprendizaje del estudiantado, al verse estas asociadas a actitudes más positivas, en contraposición con otros estudiantes que no han tenido otros acercamientos de índole académico (García, Fallas y Romero, 2015).

Se perfila como necesidad, el estudio de las percepciones y concepciones hacia las estadísticas, ya que como señala Colón (2012) los cambios en la instrucción y el aprendizaje de esta asignatura, están relacionados con el éxito académico del estudiantado que ha tomado cursos introductorios de estadística y donde a su vez, se han considerado las diferentes características demográficas y académicas de los mismos. Esta acotación es de suma importancia, ya que los estudiantes universitarios, como es el caso de la carrera de psicología, presentan conocimientos teóricos y operativos en matemáticas que son muy heterogéneos (Comas, Martins, Estrada y Nasicmento, 2017). Por ende, se ha visualizado en otras experiencias, estudios donde se han visto implicadas las experiencias, motivaciones, dificultades y temores respecto de la estadística en estudiantes de psicología, lo cual desencadena en la reflexión de cómo crear mejores estrategias 
didácticas que faciliten el intercambio de conocimiento entre docente-estudiante-docente (Escalante, Repetto y Mattinello, 2012).

Es de basto conocimiento para la Universidad Latina de Costa Rica, que en los cursos donde se ven implicados el contenido y el uso de las matemáticas y las estadísticas, es donde se ha llegado a presentar la mayor proporción de estudiantes desertores, lo cual invita a la reflexión de la metodología de clase y de los recursos utilizados en la enseñanza, para lograr una mejor relación entre estudiante-docente-asignatura. Y es que la concepción de dificultad o poco manejo, puede estar asociado con las experiencias previas y emociones hacia la materia, incluso antes de iniciar su formación en la misma, tal y como lo resalta De Miguel (2015).

Las actitudes han sido considerablemente estudiadas en los campos académicos y por esa razón, precisar en su definición resulta difícil dado los enfoques y significados que se le han otorgado. De ahí que, se ha investigado en disciplinas de forma no generalizada, para poder ahondar en las temáticas que corresponden a cada área del saber. Ejemplo de esto, es posible enunciar a Facundo, Auné y Horacio (2018), los cuales mencionan la importancia de crear investigaciones acordes a las carreras, por las diferencias cognitivas que existen entre ellas, tal es el caso de la capacidad de abstracción y el razonamiento lógico, lo que conlleva a concebir a las actitudes como un constructo que integra las experiencias académicas, los conocimientos previos en estadística y matemática, la relación con los docentes y con los mismos contenidos de la materia. Por otra parte, el desarrollo del pensamiento estadístico ha sido obstaculizado por distintas razones, como lo aclaran Carreño y Mayorga (2017) al verse implicados: “...la gestión de datos, la captura/manipulación de datos, el software estadístico y los tipos de investigación científica" (p. 349). De esa manera, se visualizan escenarios de estudio que integren en la práctica profesional y académica, el uso de las estadísticas, que ayuden a promover un aprendizaje de mayor provecho.

Para esta investigación la actitud se va a definir como la combinación de creencias y emociones que predisponen a una persona a responder positiva o negativamente ante otros individuos, objetos o instituciones (Coon, 2005). Asimismo, De Miguel (2015) anota que: "La actitud concreta hacia la estadística es una tendencia que se forma a lo largo del tiempo y como consecuencia de las emociones y los sentimientos experimentados en el contexto del aprendizaje de las matemáticas y la estadística" (p.355). 
Para esta investigación, se estudian las actitudes de los estudiantes universitarios hacia la estadística, a razón de seis dimensiones (Escala actitudes hacia las estadísticas de Estrada, 2002) dividas en los siguientes componentes, según Salinas y Mayén (2016):

1) Componentes sociales. Son las actitudes que tienen las personas, como ciudadanos, hacia la estadística en términos de su valor social y cultural.

2) Componentes educativos. Valoran la utilidad percibida de esta materia dentro de la malla curricular y la dificultad según el entrevistado.

3) Componentes instrumentales. Se refieren a la valoración de la estadística como una herramienta de utilidad para comprender otras materias o lograr razonamientos que van más allá de la estadística en sí misma.

4) Componentes afectivos. Valoran el nivel de agrado e interés que sienten las personas hacia la estadística.

5) Componentes cognitivos. Es importante diferenciar este componente del anterior, porque aun cuando una persona sienta agrado por una materia podría encontrarla difícil. Esa percepción de dificultad es la que se busca medir con el componente cognitivo.

6) Componente comportamental. Valora la tendencia de las personas a utilizar la estadística cuando lo consideran útil.

Se tratará de establecer además una relación entre las predisposiciones favorables y desfavorables, que se presentan entre las Facultades de Ciencias Exactas, Ciencias de la Salud y Ciencias Sociales de la Universidad Latina de Costa Rica, con respecto a esta materia de estudio.

En el bagaje internacional del estudio de las actitudes hacia las estadísticas, existen diversas investigaciones que muestran estudiantes y docentes hacia la probabilidad y estadística (Estrada, Bazán y Aparicio, 2013; Estrada, Batanero y Fortuny, 2004; Salinas y Mayén, 2016). De la misma forma, la inclusión del estudio de las actitudes en contextos matemáticos universitarios, ha sido de interés en otros estudios (PérezTyteca, 2012).

La psicología como ciencia disciplinar, ha sido de igual manera objeto de estudio y para esto, se ha considerado la exposición previa a contenidos estadísticos y resultados en la materia de la asignatura en la carrera de Psicología (Bologna y Vaiman, 2013).

Además, en ese mismo eje temático, se han implementado diversas estrategias de medición (Mayén y Salinas, 2016; Torres, Aparicio, Bazán, Abdounur, 2015; Vargas, Bazán, Aparicio, 2013) que han hecho uso de escalas, como la Escala de Actitudes hacia 
la Estadística (EAEE), donde se ha comprobado evidencia de la multidimensionalidad de las escalas y diferencias significativas por especialidad.

Por último, en Costa Rica las investigaciones realizadas en relación al campo de las estadísticas, se enfocan en las áreas de matemáticas en general. Tal es el caso del estudio de Gamboa y Moreira (2017) al estudiar las actitudes y creencias respecto a las matemáticas, donde se determina que las actitudes y el ambiente de clase favorecen el proceso de aprendizaje. Sin embargo, no se encontraron estudios específicos sobre la actitud hacia la estadística en este país.

Para la investigación, se plantearon los siguientes objetivos.

Objetivo general

Determinar las actitudes que tienen estudiantes de la Universidad Latina de Costa Rica, Sede San Pedro respecto a la estadística como materia de estudio y como herramienta para el análisis y comprensión de situaciones cotidianas.

Objetivos específicos

1. Contrastar en las áreas cognitiva, afectiva, comportamental, así como social, educativa e instrumental, las actitudes que poseen los participantes hacia la estadística, para discutir los componentes actitudinales que se ven implicados en el proceso de enseñanza y aprendizaje de esa materia.

2. Examinar las tendencias favorables y desfavorables, que poseen los universitarios hacia las estadísticas según la facultad, para valorar si existen diferencias actitudinales entre las disciplinas de estudio.

3. Comparar las actitudes de estudiantes hacia la estadística según la facultad a la que pertenecen, con el fin de determinar las variaciones entre las áreas disciplinares.

Analizar las propiedades psicométricas de la Escala de Actitudes hacia la Estadística.

\section{Método}

El estudio realizado es de tipo no experimental, transversal descriptivo. Se trabajó con los estudiantes que ya estaban matriculados, según cada carrera. Para cumplir con los plazos del estudio, se recolectaron los datos en un periodo de un cuatrimestre (mayoagosto del 2017), abarcando los cursos activos de estadística para todas las carreras. Es decir, se utilizaron grupos naturales. Algunos de estos grupos estudiaron estadística 
asistidos por computadora, mientras que otros aprendieron los procedimientos de forma manual.Investigación descriptiva-transversal

\section{Participantes}

Se trabajó el estudio con una muestra voluntaria de 303 estudiantes de las facultades de Ciencias Exactas (46.5\%), Ciencias de la Salud (45\%) y Ciencias Sociales (8.5\%) de la Universidad Latina de Costa Rica. El instrumento se diseñó para ser aplicado a todos los estudiantes del periodo enunciado, los datos que se reportan responden a las personas que accedieron a participar en la investigación y a los cuestionarios que se entregaron de forma completa.

La distribución por sexo de los participantes fue de $60 \%$ mujeres y $40 \%$ hombres, con una edad media de 22,38 años ( $s=4.8$ años). La media del cuatrimestre que cursaban los estudiantes, al momento de recolectarse los datos fue de 4.8 cuatrimestres cursados ( $s=2.6$ cuatrimestres), indicando que el promedio de participantes se encontraba iniciando su segundo año de carrera, lo cual los clasificaría como estudiantes poco avanzados, ya que durante el primer año la mayoría de los cursos que se les impartes son de servicio. En relación a la nacionalidad, predominan los estudiantes costarricenses $(n=281)$.

Para el tipo de colegio del cual obtuvieron el título de educación media, se contabilizaron 141 personas provenientes de colegios públicos, 135 de colegios privados, 26 de institutos (bachillerato por madurez) y un estudiante que se abstuvo de responder. El 73\% aseguró no haber cursado estadística antes, a pesar de que la materia se encuentra en los programas de primaria y secundaria.

El tamaño de la muestra, se justifica dado que la Escala de Actitudes hacia la Estadística EAEE (Estrada, 2002), consta de 25 ítems y se añadieron 8, algunos autores tales como Carretero y Pérez (2005), recomiendan tener como mínimo entre 5 y 10 individuos por cada ítem en la escala.

\section{Instrumento}

Se utilizó la Escala de Actitudes hacia la Estadística (EAEE) (Estrada, 2002) compuesta por 25 ítems (14 afirmativos y 11 negativos) que mide las actitudes hacia el área de interés en cinco puntajes: 1. Completamente en desacuerdo, 2. Parcialmente en 
desacuerdo, 3. Ni de acuerdo ni en desacuerdo, 4. Parcialmente de acuerdo y 5. Completamente de acuerdo.

Para el análisis de resultados, estas puntuaciones se invirtieron en los 11 ítems negativos que presenta. Después de invertir la escala, se tiene que las puntuaciones más altas indicarán actitudes positivas hacia la estadística y las más bajas indicarán lo contrario (Ver Anexo 1).

Ahora bien, se anotaron otras variables a la escala de Estrada (2002) que fueron: sexo, edad, nacionalidad, carrera cursada, cuatrimestre que cursan, tipo de colegio donde se obtuvo el título de educación media, año en que se finalizan los estudios de educación secundaria y si se tienen estudios en estadística previos a los universitarios.

A esta escala se le han realizado estudios de evaluación de las propiedades psicométricas (Estrada, Bazán y Aparicio, 2013), resultando ser adecuados y obteniéndose una escala multidimensional. Según estas evaluaciones algunos de los ítems sugieren ser eliminados. Sin embargo, a nivel de Costa Rica, esta escala no se ha analizado y se decide hacer el análisis psicométrico de los 25 ítems para obtener una valoración inicial y exploratoria de la misma en el contexto costarricense. Los resultados obtenidos para los 303 estudiantes y las principales conclusiones sobre la escala se presentan en el apartado de resultados.

\section{Procedimiento}

La aplicación del instrumento estuvo a cargo de dos profesoras de la Facultades de Ingeniería y TIC’S y Salud. Se visitaron diferentes grupos en horarios alternos de las carreras de Administración de Empresas, Contabilidad, Psicología, Enfermería, Farmacia, Trabajo Social, Ingenierías, entre otras. El estudio se presentó a los estudiantes como una investigación para indagar la actitud de los mismos hacia la estadística como materia de estudio y herramienta para el análisis y comprensión de situaciones cotidianas. A los estudiantes, en cada clase visitada, se les hizo lectura de un documento donde se les indicaba que se asegurará la confidencialidad y anonimato de las respuestas, así como la posibilidad de interrumpir la administración de la escala cuando se considerara conveniente. 


\section{Análisis estadístico}

Se implementaron como técnicas de análisis estadístico, el ANOVA de un factor, correlaciones bivariadas (Pearson), pruebas de independencias de grupos, como pruebas $t$ y de contraste de variables categóricas, ji-cuadrado, considerando como referencia el valor de $p=0.05$.

Además, se analizaron las propiedades psicométricas de la EAEE (Estrada, 2002) mediante un Análisis Factorial Exploratorio bajo el Método de Componentes Principales y con rotación Varimax, así como consistencia interna con el Alfa de Cronbach.

\section{Resultados}

De las variables demográficas del estudio, la edad de los participantes correlaciona de forma positiva y en una magnitud media-alta con la actitud favorable que se presenta hacia la materia de estadística $(r=0.72, p=0.02)$, lo cual permite inferir que existe mayor madurez en el proceso de aprendizaje, mayor nivel de conciencia de la utilidad de la misma y en la resolución y comprensión de problemas cotidianos. Esto se puede demostrar, de forma más concisa con las siguientes correlaciones (Tabla 1):

\begin{tabular}{|c|c|c|}
\hline \multicolumn{3}{|c|}{$\begin{array}{l}\text { Tabla } 1 \\
\text { Correlaciones obtenidas entre la edad de los participantes e ítems de la EAEE }\end{array}$} \\
\hline Ítem & $r$ & $p$ \\
\hline $\begin{array}{l}\text { 3. A través de la estadística se puede manipular la } \\
\text { realidad. }\end{array}$ & 0.86 & 0.01 \\
\hline $\begin{array}{l}\text { 4. La estadística es fundamental en la formación básica } \\
\text { del futuro ciudadano }\end{array}$ & 0.53 & 0.03 \\
\hline $\begin{array}{l}\text { 10. Me gusta la estadística porque me ayuda a comprender } \\
\text { más profundamente la complejidad de ciertos temas. }\end{array}$ & 0.71 & 0.02 \\
\hline $\begin{array}{l}\text { R23.Si pudiera eliminar alguna materia sería la } \\
\text { estadística. }\end{array}$ & 0.99 & 0.00 \\
\hline
\end{tabular}

Nota: Elaboración propia a partir de los resultados de la investigación. El enunciado 23, corresponde a un ítem invertido.

Los enunciados presentados en la Tabla 1, muestran que la edad de los universitarios se relaciona estrechamente con la compresión de temas en el área estadística y más allá de eso, que su aprovechamiento en el ámbito académico puede ser mayor, en la medida que se van desarrollando mayores acercamientos a la materia y de la importancia que le confieren a la inclusión de estos cursos en los planes de estudio de las carreras. 
Se realiza énfasis en la edad de los universitarios, al identificar el año de finalización de estudios secundarios entre el periodo 1988 al 2009, representando el 19\% de la muestra y en el periodo 2010 al 2016, ubicando al $81 \%$ de los participantes.

\section{Análisis descriptivo de las dimensiones de la EAEE}

Interesó resaltar las dimensiones que obtuvieron los valores más altos de la EAEE (Estrada, 2002). En este caso, predominaron las áreas afectiva, social, educativa y cognitiva (Tabla 2). Estos datos, evidencian que los estudiantes tienden a elaborar conceptualizaciones acerca de su formación en estadística, a partir de experiencias académicas pasadas, necesidad de informarse de la materia para comprender situaciones cotidianas o reportajes de medios de comunicación, sentimientos y emociones que les evocan valoraciones favorables y desfavorables hacia las ciencias exactas.

\section{Tabla 2}

Estadísticas descriptivas por cada dimensión que integra la EAEE (Estrada, 2002)

\begin{tabular}{lcccc}
\hline Dimensión & $\bar{x}$ & $s$ & Mínimo & Máximo \\
\hline Social & 30,60 & 4,83 & 14 & 40 \\
Educativa & 28,99 & 6,44 & 11 & 45 \\
Instrumental & 23,23 & 5,07 & 11 & 35 \\
Afectivo & 32,12 & 6,92 & 16 & 48 \\
Cognitivo & 26,83 & 3,89 & 16 & 36 \\
Comportamental & 22,99 & 4,75 & 10 & 34 \\
\hline Nota: Elaboración propia a partir de los resultados de la investigación.
\end{tabular}

Se realizó también un análisis de varianza de una vía (ANOVA), con el fin de identificar los ítems con puntuaciones significativamente diferentes. Esto permite determinar los ítems de puntuaciones significativamente altas y los de puntuaciones significativamente bajas.

Del análisis, resultó que los dos ítems con valores más bajos son el 20 (Me gusta hacer problemas cuándo uso la estadística) y el 22 (A menudo explico a mis compañeros problemas de estadística que no han entendido). La primera de estas preguntas se cataloga dentro del ámbito afectivo, la segunda se cataloga como comportamental. Ambas tienen un promedio inferior a 3 puntos, lo que se considera como actitudes negativas hacia la estadística.

Los ítems con los puntajes más altos fueron el 19 (La estadística sólo sirve para la gente de ciencias) y el 25 (Evito las informaciones estadísticas cuando las leo). A ambos ítems, se les invirtió los puntajes por contener reactivos negativos. Para el ítem 25, una puntuación alta indica que los estudiantes no evitan la información estadística cuando la 
encuentran. El ítem 19, denota que los estudiantes están conscientes de la necesidad de aprender la estadística en cualquier carrera ya sea de ciencias o no. De ahí, que surge la pregunta de si esta afirmación es consistente en todas las respuestas. Por esta razón se realizaron análisis de varianza de una vía, para cada pregunta, comparando las respuestas por facultad, por lo que las carreras estudiadas se agruparon de la siguiente forma: Ciencias de la Salud, Ciencias Sociales, Ciencias Exactas.

En la mayoría de las dimensiones de la escala, no se presentaron diferencias por facultad, pero en algunos ítems estas diferencias sí existen. Tal es el caso de la pregunta 5 (Uso la estadística para resolver problemas de la vida cotidiana), los resultados del ANOVA muestran que los estudiantes de Ciencias Exactas están a favor de esta afirmación en mayor medida que los estudiantes de Ciencia de la Salud.

La siguiente pregunta en la que se encontró diferencia por facultad fue en la 7 (Me divierto en las clases que se explica estadística), en este caso de nuevo entre los estudiantes de Ciencias de la Salud se ven calificaciones significativamente inferiores a las de estudiantes de ciencias exactas. Un comportamiento similar se percibe en las preguntas 12 (Encuentro interesante el mundo de la estadística), 14 (Utilizo poco la estadística fuera de la Universidad), 17 (La estadística es fácil) y 20 (Me gusta hacer problemas cuando uso la estadística). En todos estos ítems, los estudiantes de ciencias exactas muestran puntuaciones más altas que los de la Facultad de Ciencias de la Salud.

En la pregunta 16 (Me apasiona la estadística porque ayuda a ver los problemas objetivamente) y la pregunta 24 (La estadística ayuda a tomar decisiones más documentadas), la diferencia se vio entre ciencias exactas y ciencias sociales. En ambos casos la puntuación para las carreras de ciencias exactas fue superior.

Asimismo, al contrastar la variable "Año en que se finalizaron estudios de secundaria" con "facultad a la que pertenecen los estudiantes", se encontró que Ciencias de la Salud a diferencia de Ciencias Sociales y Exactas, reportó una fecha de graduación de mayor antigüedad que las demás carreras, lo cual podría influir en las reacciones del estudiantado hacia materias que contemple las matemáticas, o en este caso particular a las estadísticas debido a que este segmento de los participantes no recibieron un énfasis en estadística como parte de los planes educativos de secundaria.

En general se obtuvo con la investigación, que las puntuaciones más altas se concentran en los estudiantes de Ciencias Exactas. La justificación que se supone para esta situación, es que este grupo de estudiantes tienen mayor afinidad por las matemáticas, dada la selección previa de carrera que ha hecho por las aptitudes de estos. Es decir, se 
trata de estudiantes que en sus planes de estudios tienen cursos de cálculo, álgebra, ecuaciones o similares. Por lo que, son estudiantes que han estudiado o planean estudiar diferentes ramas de las matemáticas y las matemáticas aplicadas. Esto no ocurre con los estudiantes de la Facultad de Ciencias de la Salud ni los de Ciencias Sociales, dado que las mallas curriculares de estas carreras no contemplan esta concentración tan exhaustiva de cursos relacionados con las matemáticas.

\section{Comparación de resultados con otros estudios}

Interesa comparar los resultados obtenidos en ULAT con otros estudios similares realizados en otras latitudes. En España, un estudio similar se aplicó a estudiantes universitarios de Psicología (Comas, Martins, Nacimiento y Estrada, 2017). Los ítems utilizados en dicho estudio coinciden con los empleados en ULAT por lo que la comparación es posible uno a uno. Se comparan los resultados del estudio español (108 respuestas) con los resultados de $\operatorname{los} 87$ estudiantes de ULAT, que respondieron al cuestionario y que pertenecían a la carrera de psicología.

Según señala el estudio español, “oficialmente la Estadística está presente en los diferentes niveles educativos (Primaria/ESO/ Bachillerato o FP)" (Comas, Martins, Nacimiento y Estrada, 2017, p.485), este es un caso similar al de Costa Rica, en donde los programas educativos de matemática incluyen el estudio de conceptos básicos de estadística en primaria y secundaria. Sin embargo, en el estudio español, un 33\% de los estudiantes indicaron nunca haber estudiado estadística, previo a su formación universitaria. Para los estudiantes de ULAT, un $72 \%$ indica lo mismo. Esta diferencia puede deberse a la reciente inclusión explícita de la estadística en los programas de estudio costarricenses.

Con respecto a la escala de Estrada (2002), se hizo una comparación para detectar, si se presentan respuestas significativamente distintas entre costarricenses y españoles. Las preguntas utilizadas en ambos estudios son idénticas y se han invertido las respuestas de los ítems con negaciones en ambos casos, así es posible hacer una comparación ítem a ítem.

Al aplicar una prueba de hipótesis para medias, se encuentran diferencias significativas en las respuestas de algunos ítems (Tabla 3). Sin embargo, a pesar de que las diferencias existen, en ninguno de los casos la diferencia es tan grande como demostrar un desacuerdo sobre el ítem por parte de los estudiantes. Es decir que, hay diferencias en 
los valores de las medias, pero igual acuerdo porque, en general, se da una respuesta positiva (o negativa) al ítem en ambas muestras.

La diferencia más grande encontrada se da en el ítem 18 (Me entero más de los resultados de las elecciones cuando aparecen representaciones gráficas), donde la media española fue de 4.07 y la costarricense de 3.40. Se puede considerar que los estudiantes españoles brindaron una respuesta positiva en este ítem, mientras que los costarricenses fueron neutrales. Llama la atención que entre los estudiantes españoles este ítem fue el tercero mejor puntuado, mientras que entre los costarricenses no se encuentra en una posición de igual importancia.

Tabla 3

Comparación de medias estudio ULAT y Comas, Martins, Nacimiento y Estrada

\begin{tabular}{|c|c|c|c|c|c|c|}
\hline \multirow[b]{3}{*}{ Ítem } & \multicolumn{5}{|c|}{ Nuevas agrupaciones según resultados obtenidos } & \multirow{3}{*}{ Valor $p$} \\
\hline & \multirow[b]{2}{*}{ Texto del ítem } & \multicolumn{2}{|c|}{$\begin{array}{l}\text { Estadísticos } \\
\text { España }\end{array}$} & \multicolumn{2}{|c|}{$\begin{array}{l}\text { Estadísticos } \\
\text { ULAT }\end{array}$} & \\
\hline & & $\bar{x}$ & $s$ & $\bar{x}$ & $s$ & \\
\hline E4 & $\begin{array}{l}\text { La estadística es fundamental en } \\
\text { la formación básica del futuro } \\
\text { ciudadano. }\end{array}$ & 3.09 & 0.85 & 3.49 & 0.97 & 0.003 \\
\hline E18 & $\begin{array}{l}\text { Me entero más de los resultados } \\
\text { de las elecciones cuando } \\
\text { aparecen representaciones } \\
\text { gráficas. }\end{array}$ & 4.07 & 0.83 & 3.40 & 1.17 & 0.004 \\
\hline E20 & $\begin{array}{l}\text { Me gusta hacer problemas } \\
\text { cuando uso la estadística. }\end{array}$ & 2.77 & 1.00 & 2.17 & 0.15 & 0 \\
\hline E22 & $\begin{array}{l}\text { La estadística ayuda a tomar } \\
\text { decisiones más documentadas. }\end{array}$ & 2.68 & 1.21 & 2.24 & 1.32 & 0.017 \\
\hline
\end{tabular}

Fuente: Elaboración propia.

Al revisar los tres ítems con las puntuaciones más altas en cada estudio, se encontró que tanto costarricenses como españoles coinciden en los ítems 19 y 21 ( $L a$ estadística sólo sirve para la gente de ciencias y La estadística no sirve para nada). Esto demuestra que, en ambos casos, los estudiantes comprenden la importancia de la estadística y su necesidad en otras áreas además de las ciencias.

\section{Análisis de las propiedades psicométricas de la escala}

Para la valoración de las propiedades psicométricas de la EAEE (Estrada, 2002), se realizaron pruebas de confiabilidad (consistencia interna) y validez (análisis factorial exploratorio). Esto con el fin de determinar que la escala fuera válida, de lo contrario los resultados obtenidos no representarían datos poblacionales. 
Respecto a la confiabilidad, se obtuvo un alfa de Cronbach alto $(\alpha=0.87)$, lo que demuestra que la escala por puntuación total es confiable. Para aumentar dicha puntuación, fue necesario eliminar los ítems 3 y 14, ya que no puntuaban correctamente dentro de la escala.

En Tabla 4, se muestra por cada dimensión los resultados obtenidos en el análisis de fiabilidad de los ítems.

Tabla 4

Estadísticas descriptivas por cada dimensión que integra la EAEE (Estrada, 2002)

\begin{tabular}{lc}
\hline Dimensión & $\alpha$ \\
\hline Social & 0.63 \\
Educativa & 0.80 \\
Instrumental & 0.76 \\
Afectivo & 0.83 \\
Cognitivo & 0.63 \\
Comportamental & 0.61 \\
\hline Nota: Elaboración propia a partir de los resultados de la investigación. & \\
\hline
\end{tabular}

Análisis factorial exploratorio, con extracción de factores por medio del método de componentes principales

Como se ha mencionado la escala cuenta con seis agrupaciones teóricas: afectiva, comportamental, cognitiva, social, educativo e instrumental. No obstante, no necesariamente todos los ítems de una agrupación teórica fueron puntuados de manera similar por los estudiantes. Por esta razón se decide hacer un análisis de covarianzas. Este análisis permite determinar cuáles ítems son puntuados de manera similar por los estudiantes y por lo tanto determinar qué agrupaciones son las que ellos perciben en términos del uso y aprendizaje de la estadística.

$\mathrm{Al}$ aplicar las pruebas de esfericidad de Bartlett y calcular la medida de adecuación muestral KMO, se obtuvieron los siguientes resultados (Tabla 5).

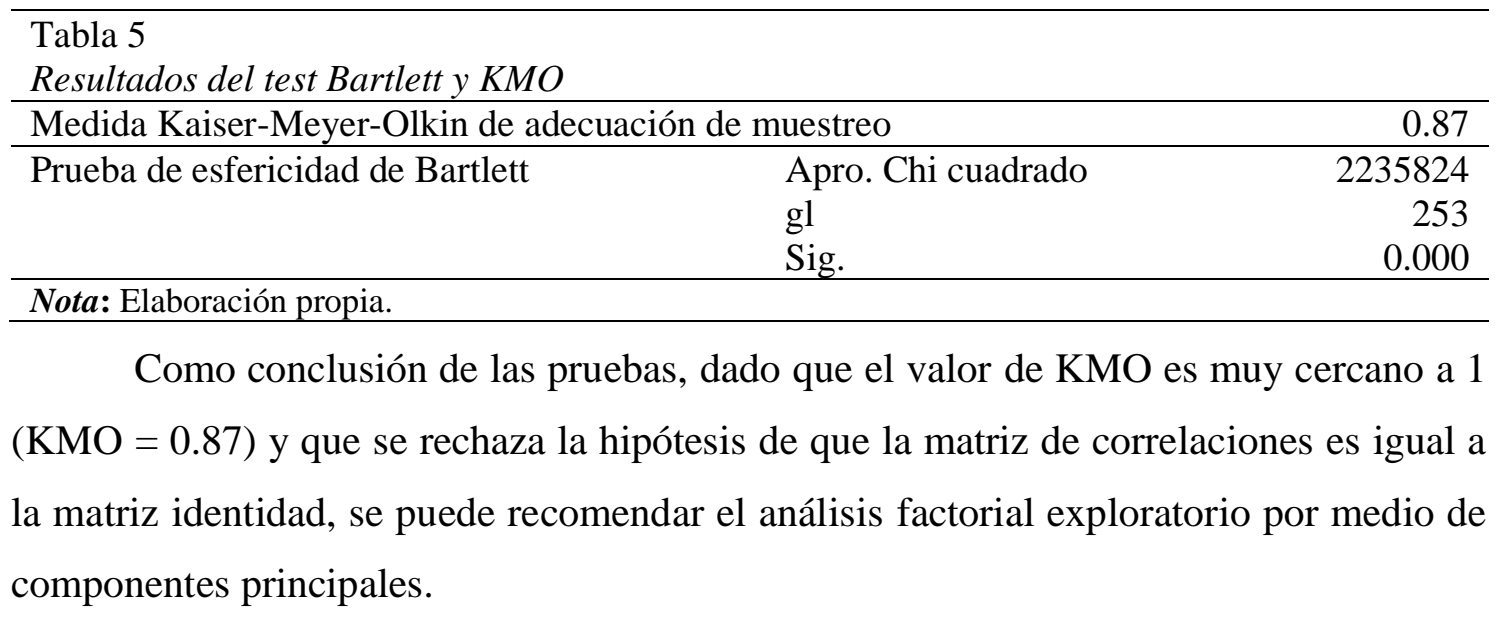


Al extraer los factores por el método de componentes principales y realizando una rotación Varimax, fue posible extraer 6 factores que explican el $61 \%$ de la varianza total de ahí que se pueden agrupar los 23 ítems (que sí discriminan) del instrumento, en los siguientes grupos (Tabla 6):

Tabla 6

Comportamiento de factores en términos de varianza explicada

\begin{tabular}{|c|c|c|c|}
\hline \multirow[b]{2}{*}{ Ítem } & \multicolumn{3}{|c|}{ Nuevas agrupaciones según resultados obtenidos } \\
\hline & 1 & 2 & 3 \\
\hline E2 & .512 & & \\
\hline E4 & 636 & & \\
\hline E5 & .571 & & \\
\hline E7 & .598 & & \\
\hline E8 & & .786 & \\
\hline E10 & .709 & & \\
\hline E12 & .720 & & \\
\hline E13 & 639 & & \\
\hline E16 & .654 & & \\
\hline E17 & & .795 & \\
\hline E18 & 441 & & \\
\hline E20 & .557 & & \\
\hline E22 & & 680 & \\
\hline E24 & .450 & & \\
\hline r1 & & & 406 \\
\hline r6 & & & .537 \\
\hline r9 & & & 415 \\
\hline r11 & & .545 & \\
\hline r15 & & & .564 \\
\hline r21 & & & .735 \\
\hline r23 & .537 & & .512 \\
\hline r25 & & & 490 \\
\hline r19 & & & .651 \\
\hline
\end{tabular}

Nota: Los ítems con la anotación de "r" indican los que poseen puntajes inversos (5=Completamente en desacuerdo, $4=$ Parcialmente en desacuerdo, $3=\mathrm{Ni}$ de acuerdo ni en desacuerdo, 2=Parcialmente de acuerdo, 1=Completamente de acuerdo).

El análisis de covarianzas (con rotación varimax) permite determinar los ítems que se discriminan de cada dimensión, según las respuestas obtenidas de los estudiantes.

Esto permite hacer una comparación entre las agrupaciones teóricas y las tres nuevas agrupaciones que se crean con base en los resultados que se tienen.

En el Tabla 7, se muestran los ítems divididos en las tres escalas antropológicas originales, así como la agrupación que se ha detectado con el análisis de covarianza. 
Tabla 7

Agrupaciones por covarianzas en comparación con las dimensiones antropológicas de la escala original

\begin{tabular}{cccccc}
\hline $\begin{array}{c}\text { Escala Original } \\
\text { (Afectivo) }\end{array}$ & $\begin{array}{c}\text { Escala } \\
\text { ULAT }\end{array}$ & $\begin{array}{c}\text { Escala Original } \\
\text { (Cognitivo) }\end{array}$ & $\begin{array}{c}\text { Escala } \\
\text { ULAT }\end{array}$ & $\begin{array}{c}\text { Escala Original } \\
\text { (Comportamental) }\end{array}$ & $\begin{array}{c}\text { Escala } \\
\text { ULAT }\end{array}$ \\
\hline 1 & 2 & 2 & & 5 & 1 \\
7 & 7 & $\mathbf{3}$ & & 8 & 6 \\
10 & 10 & 4 & 11 & 14 & 9 \\
11 & 4 & 6 & 8 & 15 & 15 \\
12 & 12 & 9 & 17 & 19 & 19 \\
13 & 13 & 17 & 22 & 22 & 21 \\
16 & 16 & 21 & & & 23 \\
20 & 20 & 24 & & & \\
23 & 23 & & & & \\
25 & 5 & & & & \\
& 18 & & & & \\
\end{tabular}

Nota: Los ítems 3 y 14 no discriminaron en ninguna categoría, por esta razón aparecen en la escala original pero no en las agrupaciones de los resultados de la Universidad Latina (ULAT).

Se denota que, con respecto a la escala original, la nueva agrupación muestra variaciones. La mayor cantidad de concurrencias se dieron entre la dimensión afectiva y la primera agrupación por covarianzas. En este caso, los resultados obtenidos se agrupan de forma similar a como se muestran en la escala original. Pese a esto, para las dimensiones cognitiva y comportamental, los ítems que la escala original presenta en estas categorías, no coinciden con las otras dos agrupaciones obtenidas según los resultados de los estudiantes de la ULAT. Además, hay ítems de otras escalas que, según los resultados de los estudiantes, pasarían a formar parte de la escala afectiva.

La interpretación que se puede hacer de este resultado es que algunos de los ítems con los que se esperaba medir actitud cognitiva o comportamental, recibieron respuestas similares a los ítems que medían actitudes afectivas. Es decir que, para los estudiantes la principal dimensión en cuánto a su percepción de la estadística, es la afectiva y desde esta dimensión es que se hacen evaluaciones que se asumen de tipo cognitivo y comportamental. Los participantes se guían por sus emociones, para evaluar a la estadística y no tanto por las aplicaciones de ésta.

Dado que la escala original agrupa los ítems tanto de forma antropológica como sociocultural, se procede a revisar las concurrencias entre las categorías obtenidas y las teóricas, pero esta vez se hace para las dimensiones social, educativa e instrumental. La Tabla 8, resume las concomitancias para las tres dimensiones. 
Tabla 8

Agrupaciones por covarianzas en comparación con las dimensiones socioculturales de la escala original

\begin{tabular}{cccccc}
\hline $\begin{array}{c}\text { Escala original } \\
\text { (Social) }\end{array}$ & $\begin{array}{c}\text { Escala } \\
\text { ULAT }\end{array}$ & $\begin{array}{c}\text { Escala original } \\
\text { (Educativo) }\end{array}$ & $\begin{array}{c}\text { Escala } \\
\text { ULAT }\end{array}$ & $\begin{array}{c}\text { Escala original } \\
\text { (Instrumental) }\end{array}$ & $\begin{array}{c}\text { Escala } \\
\text { ULAT }\end{array}$ \\
\hline 1 & 1 & 4 & & 5 & 5 \\
2 & 6 & 6 & & 10 & 10 \\
9 & 9 & 7 & & 13 & 13 \\
11 & 23 & 8 & 8 & 16 & 16 \\
18 & 15 & 12 & 11 & 20 & 20 \\
19 & 19 & 15 & & $\mathbf{3}$ & 7 \\
21 & 21 & 17 & 17 & 24 & 24 \\
25 & 25 & 22 & 22 & $\mathbf{1 4}$ & 4 \\
& & 23 & & & 2 \\
& & & & & 18 \\
\hline
\end{tabular}

Nota: Los ítems 3 y 14 no discriminaron en ninguna categoría, por esta razón aparecen en la escala original pero no en las agrupaciones de los resultados de la Universidad Latina (ULAT).

De nuevo se observa que una de las dimensiones originales, la educativa no se conformó de manera contundente cuando se emplearon las covarianzas. Algunos ítems que se consideraban como sociales o educativos, se han agrupado dentro de la dimensión instrumental, indicando que, para los estudiantes, el uso que se le dé a la estadística abarca tanto el uso dentro de las aulas tanto como en la sociedad.

\section{Discusión}

En general se ven actitudes positivas hacia la estadística entre estudiantes de Ciencias Exactas con más frecuencia que entre Ciencias de la Salud y Ciencias Sociales, lo cual llega a demarcar prejuicios sobre la comprensión y aptitud hacia esta materia y por ende tal y como lo expresa León y Vaiman (2013) favorece que el compromiso con el estudio sea inferior y no se profundice en los contenidos de dificultad mayor. Específicamente en las carreras de psicología y educación, se han presentado vinculaciones emocionales poco favorables hacia las matemáticas y las estadísticas, por lo que el desarrollo de investigaciones con metodologías mixtas, podrían acercar a una realidad más precisa del acontecer de estas poblaciones universitarias, al considerar elementos propios de cada carrera y de esa manera, la posibilidad de profundizar en las variables que incluyen estos procesos de formación (Facundo, Auné y Horacio, 2018).

Del análisis de covarianzas se determinó que las agrupaciones teóricas, no corresponden con las nuevas agrupaciones que se crean a partir de los resultados 
obtenidos. Las dimensiones con más ítems, según este análisis, fueron la afectiva y la instrumental, mientras que resultaron con menos ítems la cognitiva y la educativa. Esto indica que los estudiantes respondieron a diversos ítems con respecto al grado de interés que sienten hacia la estadística y la utilidad que ven en ella, incluso cuando se trataba de ítems que buscaban medir su actitud como ciudadanos o la dificultad que encontraban en la materia. Esto sugiere que la escala necesita adaptaciones para ser aplicada en diferentes latitudes. Ya sea porque la redacción de los ítems es variable en sus interpretaciones o porque los estudiantes se acercan de formas diferentes al estudio de la estadística; lo que se encontró sugiere que no siempre notan diferencias entre una pregunta asociada al nivel de dificultad de una materia y otra asociada al nivel de agrado que sienten por la misma materia, esto por poner un ejemplo. La escala debería analizarse en una prueba piloto y ajustarse si fuera necesario para asegurar que los entrevistados noten las diferencias entre las diferentes preguntas y se logre medir todas las dimensiones de forma semejante.

En relación a los resultados de las ANOVAS, se obtiene que, para los estudiantes de Ciencias de la Salud, es menos común la aplicación de la estadística fuera de las aulas. Se determinó que, para estudiantes de ciencias exactas, la estadística aporta un factor de entretenimiento o reto positivo, situación que no se presenta entre estudiantes de Ciencias de la Salud, al exponerse percepciones desfavorables hacia esta materia.

Si bien los estudiantes indican explícitamente que la estadística no es sólo para personas de ciencias exactas (pregunta 19, una de las preguntas con puntajes positivos más altos, con promedio de 4.34), los estudiantes siguen respondiendo a otros ítems de forma diferenciada por carrera, situación que se comprobó con el uso del ANOVA. Esto indica que, a pesar de que los estudiantes afirman que la estadística tiene aplicaciones en diferentes ramas, cuando responden preguntas desde su experiencia personal, emergen diferencias que se pueden asociar a las carreras de estudio. La aseveración antes expuesta, se congruente con los hallazgos de Pérez, Aparicio, Bazán y Abdounur (2015) al haber acuerdo entre los universitarios en la importancia de la estadística y de su inclusión en su formación profesional, pese a que se sienten poco seguros en su habilidad, adecuado desempeño y aplicabilidad, lo que genera pensamientos negativos y temores en torno a esta asignatura.

Las edades de los estudiantes varían entre los 17 y 45 años, se considera que, a futuro, un estudio de este tipo podría agrupar estudiantes por rangos de edad en lugar de hacerlo por áreas de estudio. Es posible que haya diferencias en las respuestas por edades, 
porque los estudiantes mayores podrían tener un acercamiento más positivo a la estadística.

El cuestionario no cuenta con preguntas que permitan determinar la razón por la que diferentes carreras responde de diferente forma a los ítems. Como docentes, las autoras han percibido que algunos estudiantes de carreras de ciencias sociales o de ciencias de la salud, sienten que no necesitarán matemáticas en general, o estadística en particular, cuando ejerzan en sus áreas disciplinares. Por lo que sería pertinente que en estudios posteriores, se determinen las expectativas que los estudiantes universitarios tienen en relación a su desarrollo y quehacer profesional; hacer una investigación de este tipo permitiría ampliar las conclusiones de esta investigación y la relación que dichas expectativas tienen con las actitudes de los estudiantes hacia la estadística.

En cuanto a estrategias metodológicas, los resultados de la investigación demuestran que la exposición de problemas relativos a cada área disciplinar, podría aumentar la comprensión de la materia y generar un mayor dominio de la misma y consecuentemente propiciar el desarrollo de actitudes más favorables hacia esta materia de estudio. Por otra parte, incluir a estudiantes en procesos de tutoría a otros estudiantes, conllevaría a una mejor apropiación de los temas del curso y por ende, a generar enlaces académicos que contribuyan con la visión del aprendizaje de los números y no sólo del caso de la estadística.

Al comparar los resultados de ULAT con los de una universidad española en el estudio de Comas, Martins, Nacimiento y Estrada (2017), se determinó que en general los participantes de ambos estudios dieron respuestas similares. Cabe señalar que, a pesar de que en ambos países los programas de niveles anteriores al universitario, incluyen temas de estadística, hubo personas que indicaron nunca haber estudiado estadística previo a su ingreso a la universidad. Esto podría deberse, en el caso de Costa Rica, al cambio reciente en los programas de estudio para la inclusión explícita de temas estadísticos. En el caso español, se sabe que la estadística forma parte de los programas de estudio de todos los niveles y aun así el 33\% de los estudiantes negaron haber estudiado esta materia antes. Es posible que los estudiantes de ambos centros de enseñanza superior, no logren identificar la estadística dentro de los programas de matemáticas; por lo que indican no haber estudiado esta materia a pesar de haberlo hecho. Esta podría ser la base de estudios futuros para determinar si los estudiantes consideran la estadística como un tema nuevo, porque lo han olvidado o porque, teniendo los conocimientos, no los asocian con la estadística, como una disciplina del saber. Es de vital importancia considerar este 
aspecto, ya que abordar la materia como un tema nuevo, puede ser diferente a hacerlo como si se tratara de un tema conocido dentro del plano didáctico y metodológico.

Bajo esta misma idea, también se sugiere a futuro evaluar el proceso de aprendizaje mediante tareas metacognitivas en el aula, especialmente cuando se trata de matemáticas, con el fin de identificar de qué manera los estudiantes están concibiendo la materia de estadística en contraposición con la de matemáticas. Así se podrían determinar las diferencias actitudinales, conceptuales y operativas en los procesos de aprendizaje de las dos materias, lo que permitiría tener más información para analizar por qué estudiantes que tienen que haber estudiado estadística para graduarse de secundaria, indican no haberlo hecho. También es posible que el uso de computadora en el aula genere cambios en las respuestas de los estudiantes, ya que el uso de softwares estadísticos requiere de mayor abstracción y puede minimizar el aprendizaje concreto. La abstracción con software podría hacer que los estudiantes no reconozcan la estadística como una materia ya estudiada.

La escala EAEE (Estrada, 2002) se presenta de forma multidimensional y distribuye los ítems relativos al área educativa, en correspondencia con la dificultad que le atribuye el estudiantado a la materia de estadística. Las dimensiones cognitiva y afectiva, presentaron mayor equivalencia con la distribución teórica de los ítems. La escala utilizada presenta niveles medios a altos en cuanto a su confiabilidad.

Finalmente, es recomendable el desarrollo de estudios que posean relación con la autorregulación de aprendizaje de estudiantes, lo cual podría ayudar a que se puedan identificar elementos que sean parte de la motivación intrínseca de estudiantes, necesidades e intereses más afines a la población estudiantil, a la resolución de problemáticas al haber un mejor manejo de las técnicas estadística, desde la adaptación de contenidos por área disciplinar. 


\section{Referencias}

Bologna, E., \& Vaiman, M. (2013). Actitudes, experiencia previa y nivel de logro en Estadística en la carrera de Psicología. Actas de las Jornadas Virtuales en Didáctica de la Estadística, Probabilidad y Combinatoria, 91-103.

Carreño, P., \& Mayorg, J. (2017). Statistical thinking: Tool for development of nursing as a science. Avances En Enfermería, 35(3), 345-356. http://dx.doi.org/10.15446/av.enferm.v35n3.62684

Carretero, H. y Pérez, C. (2005) Normas para el desarrollo y revisión de estudios instrumentales. International Journal of Clinical and Health Psychology; 5, 521551.

Comas, C., Martins, J. A., Estrada, A., \& Nasicmento, M. M. (2017). Estudio de las Actitudes hacia la Estadística en Estudiantes de Psicología. Bolema, Rio Claro, $57,479-496$.

Colón-Rosa, H. (2012). Actitudes de estudiantes universitarios que tomaron cursos introductorios de estadística y su relación con el éxito académico en la disciplina. https://search.proquest.com/docview/1241610712?accountid=32236

Covadonga, M. (2015). Actitudes hacia la estadística de los alumnos del grado en pedagogía, educación social, y maestro de educación infantil y maestro de educación primaria de la UCM. Educación XX1, 18, 351-374. https://search.proquest.com/docview/1693713086?accountid=32236

Coon, D. (2005). Psicología. (10 Ed.) México: Editorial Thompson.

De Miguel, C. (2015). Actitudes hacia la estadística de los alumnos del grado en pedagogía, educación social, y maestro de educación infantil y maestro de educación primaria de la UCM. Educación XX1,18(2), 351-374. https://search.proquest.com/docview/1693713086?accountid=32236

Estrada, A. (2002). Análisis de las actitudes y conocimientos estadísticos elementales en la formación del profesorado. (Tesis doctoral).Universidad Autónoma de Barcelona, España.

Estrada, A., Batanero, B. y Fortuny, J. (2004). Un estudio comparado de las actitudes hacia la estadística en profesores en formación y en ejercicio. Enseñanza de las Ciencias, 2, 263-274.

Estrada, A., Bazán, E., y Aparicio, A. (2013). Evaluación de las propiedades psicométricas de una escala de actitudes hacia la estadística en profesores. Avances de Investigación en Educación Matemática, 3, 5 - 23.

Facundo, P., Auné, S., y Horacio, F.. (2018). Construcción y validación de una escala de actitud hacia la matemática para estudiantes de psicología. Universitas Psychologica, 17(4), 1-15. http://dx.doi.org/10.11144/Javeriana.upsy17-4.cvea

García, J A; Fallas, M A; Romero, A; (2015). Las actitudes hacia la estadística del estudiantado de orientación. Revista Electrónica Educare, 19, 25-41. http://www.redalyc.org/articulo.oa?id=194132805002

Gamboa, R. y Moreira, T. (2017). Actitudes y creencias hacia las matemáticas: un estudio comparativo entre estudiantes y profesores. Revista Electrónica Actualidades Investigativas en Educación, $\quad 1, \quad 1-45$. https://docs.google.com/viewerng/viewer?url=http://revistas.ucr.ac.cr/index.php/ aie/article/viewFile/27473/27672

León, E. y Vaiman, M. (2013). Actitudes, experiencia previa y nivel de logro en Estadística en la carrera de Psicología. En G. Contreras, M. Cañadas, y P. Arteaga (Eds.) Actas de las Jornadas Virtuales en Didáctica de la Estadística, Probabilidad y Combinatoria. Granada: Universidad de Granada. 
Mayén, S., Salinas, J., (2016). Un estudio comparativo de las actitudes hacia la estadística en estudiantes mexicanos de bachillerato - A comparative study of attitudes towards statistics in mexican high school studentes. Educação Matemática Pesquisa, $18(3)$. https://search.proquest.com/docview/1865236317?accountid=32236

Ministerio de Educación Pública (27 de abril 2017). Programas de estudio de matemáticas, I y II Ciclo de la Educación Primaria, III Ciclo de Educación General Básica y Educación Diversificada. Recuperado de http://www.mep.go.cr/sites/default/files/programadeestudio/programas/matemati ca.pdf

Pérez, L., Aparicio, A., Bazán, J. y Abdounur, O. (2015). Actitudes hacia la estadística de estudiantes universitarios de Colombia. Educación Matemática, 3, 111-149. http://www.scielo.org.mx/scielo.php?script=sci_arttext\&pid=S1665$\underline{58262015000300111 \& \text { lang=es }}$

Pérez-Tyteca, P. (2012). La ansiedad matemática como centro de un modelo causal predictivo en la elección de carreras (Tesis doctoral inédita). Universidad de Granada, España. http://hera.ugr.es/tesisugr/2108144x.pdf

Salinas, J. y Mayén, S. (2016). Estudio exploratorio de las actitudes hacia la estadística en estudiantes mexicanos de bachillerato. Avances de Investigación en Educación Matemática, 10, 73-90. 


\section{ANEXO 1 \\ UNIVERSIDAD LATINA DE COSTA RICA DEPARTAMENTO DE MATEMÁTICA}

Estimado estudiante: Como parte de un proceso de exploración de las actitudes hacia la estadística por parte de estudiantes universitarios, le solicito su colaboración para responder el siguiente cuestionario que responde a una investigación académica. Los datos facilitados en el cuestionario serán confidenciales y anónimos.

\section{PRIMERA PARTE. DATOS GENERALES}

Instrucciones: En esta sección se le solicita brindar su información personal, referida a diferentes aspectos. Marque con una " $X$ " la opción correspondiente o bien complete el espacio en blanco. Antes de devolver el cuestionario, revise que todas las preguntas hayan sido completadas.
1. Indique su sexo:
1. ( ) Hombre
2. ( ) Mujer

2. 2. ¿Cuál es su edad en años cumplidos?:

3. ¿Cuál es su nacionalidad?

4. 4. Anote la(s) carrera(s) que estudia:

5. Indique qué cuatrimestre de su carrera está cursando (si lleva cursos de varios cuatrimestres, indique el cuatrimestre más alto):

6. Tipo de colegio donde obtuvo su bachillerato:

7. 7. Año en que finalizó sus estudios de secundaria (colegio):

1. ( ) Público 2. ( ) Privado 3. ( ) Instituto (por madurez)

8. Antes de iniciar la universidad, indique si había estudiado estadística.

( ) 1. Nunca ( ) 2. Sí, en el colegio o escuela ( ) 3. Sí, en cursos libres ) 4. Otro. Especifique:

\section{SEGUNDA PARTE. ACTITUDES HACIA LA ESTADÍSTICA.}

A continuación se le muestra una tabla con una serie de afirmaciones. Marque con una "X" su nivel de acuerdo o en desacuerdo con cada una de esas afirmaciones. Utilice la siguiente escala.

1. Completamente en desacuerdo 2. Parcialmente en desacuerdo 3. Ni de acuerdo ni en desacuerdo. 4. Parcialmente de acuerdo 5.Completamente de acuerdo.

\begin{tabular}{|c|c|c|c|c|c|}
\hline Afirmaciones & 1 & 2 & 3 & 4 & 5 \\
\hline $\begin{array}{l}\text { 1. Me molesta la información estadística que aparece en } \\
\text { algunos programas de televisión. }\end{array}$ & & & & & \\
\hline 2. La estadística ayuda a entender el mundo de hoy. & & & & & \\
\hline 3. A través de la estadística se puede manipular la realidad. & & & & & \\
\hline $\begin{array}{l}\text { 4. La estadística es fundamental en la formación básica del } \\
\text { futuro ciudadano. }\end{array}$ & & & & & \\
\hline
\end{tabular}




\begin{tabular}{|c|c|c|c|c|c|}
\hline Afirmaciones & 1 & 2 & 3 & 4 & 5 \\
\hline $\begin{array}{l}\text { 5. Uso estadística para resolver problemas de la vida } \\
\text { cotidiana. }\end{array}$ & & & & & \\
\hline 6. En la universidad no se debería enseñar estadística. & & & & & \\
\hline 7. Me divierto en las clases que se explica estadística. & & & & & \\
\hline 8. Los problemas de estadística me resultan fáciles. & & & & & \\
\hline $\begin{array}{l}\text { 9. No entiendo la información estadística que aparece en } \\
\text { la prensa. }\end{array}$ & & & & & \\
\hline $\begin{array}{l}\text { 10. Me gusta la estadística porque me ayuda a comprender } \\
\text { más profundamente la complejidad de ciertos temas. }\end{array}$ & & & & & \\
\hline 11. Me siento intimidado ante datos estadísticos. & & & & & \\
\hline 12. Encuentro interesante el mundo de la estadística. & & & & & \\
\hline $\begin{array}{l}\text { 13. Me gustan los trabajos serios donde aparecen estudios } \\
\text { estadísticos. }\end{array}$ & & & & & \\
\hline 14. Utilizo poco la estadística fuera de la universidad. & & & & & \\
\hline $\begin{array}{l}\text { 15. En clase de estadística nunca entiendo de qué están } \\
\text { hablando. }\end{array}$ & & & & & \\
\hline $\begin{array}{l}\text { 16. Me apasiona la estadística porque ayuda a ver los } \\
\text { problemas objetivamente. }\end{array}$ & & & & & \\
\hline 17. La estadística es fácil. & & & & & \\
\hline $\begin{array}{l}\text { 18. Me entero más de los resultados de las elecciones } \\
\text { cuando aparecen representaciones gráficas. }\end{array}$ & & & & & \\
\hline 19. La estadística sólo sirve para la gente de ciencias. & & & & & \\
\hline 20. Me gusta hacer problemas cuando uso la estadística. & & & & & \\
\hline 21. La estadística no sirve para nada. & & & & & \\
\hline $\begin{array}{l}\text { 22. A menudo explico a mis compañeros problemas de } \\
\text { estadística que no han entendido. }\end{array}$ & & & & & \\
\hline 23. Si pudiera eliminar alguna materia sería la estadística. & & & & & \\
\hline $\begin{array}{l}\text { 24. La estadística ayuda a tomar decisiones más } \\
\text { documentadas. }\end{array}$ & & & & & \\
\hline 25. Evito las informaciones estadísticas cuando las leo. & & & & & \\
\hline
\end{tabular}

